\title{
The Feeling of Color: A Haptic Feedback Device for the Visually Disabled
}

\author{
Jonathan Tapson \\ Electrical Engineering \\ University of Cape Town \\ Rondebosch 7700, South Africa \\ jonathan.tapson@uct.ac.za
}

Javier Diaz

Department of Computer Architecture and Technology

Universidad de Granada

18071 Granada, Spain

jdiaz@atc.ugr.es

David Sander

Electrical and Computer Engineering

University of Maryland

College Park 20742, USA

dsander@umd.edu

\author{
Netta Gurari \\ Department of Mechanical Engineering \\ Johns Hopkins University \\ Baltimore, MD, USA \\ gurari@jhu.edu \\ Elisabetta Chicca \\ Institute of Neuroinformatics \\ UNI|ETH Zurich \\ Switzerland \\ chicca@ini.phys.ethz.ch
}

\author{
Philippe Pouliquen and Ralph Etienne-Cummings \\ Department of Electrical and Computer Engineering \\ Johns Hopkins University \\ Baltimore, MD, USA \\ retienne@jhu.edu
}

\begin{abstract}
We describe a sensory augmentation system designed to provide the visually disabled with a sense of color. Our system consists of a glove with short-range optical color sensors mounted on its fingertips, and a torso-worn belt on which tactors (haptic feedback actuators) are mounted. Each fingertip sensor detects the observed object's color. This information is encoded to the tactor through vibrations in respective locations and varying modulations. Early results suggest that detection of primary colors is possible with near $100 \%$ accuracy and moderate latency, with a minimum amount of training.
\end{abstract}

\section{INTRODUCTION}

A significant amount of information in the modern world is encoded with color. The importance of this information ranges from safety-critical (the use of orange and red as hazard warnings) to aesthetic (the expectation that garments combined into an outfit will not "clash"). The visually disabled are cut off from perception of this information. There is evidence to suggest that even those who have been unsighted since birth would appreciate adding this extra sense to their faculties [1]. In this paper we present the design and initial testing of a Chromo-Haptic Sensor-Tactor (CHST) device, which provides a sense of color to the visually disabled.
In Section II, we discuss prior work for providing sensory substitution, focusing on vibrotactile feedback to the torso. In Section III, we describe the hardware of the CHST device. In Section IV, we present the interfacing and software of the CHST device. In Section V, we discuss our initial results, describing the capabilities of our device.

\section{BACKGROUND}

In this study, we aimed to create a system that displays color information to the visually disabled. Our design goals were to render the device unobtrusive to onlookers as well as to utilize sensory systems not actively used. A clear method of providing the visually impaired with sight is through sensory substitution, e.g. haptic or auditory feedback [e.g. 18]. With our goals in mind, we chose haptic feedback for reporting of color.

There have been numerous prior attempts that investigated providing color feedback through sensory substitution [1-3]. These studies all rendered color to the visually impaired through haptic feedback. Specifically, these studies concentrated their efforts on the haptic decoding of color signals generated by a PC; they did not include an actual color sensor. In the present work we have given equal weight to the problem of color sensing, which turns out to be non-trivial 
even when performed on a point-like locality such as a fingertip.

Given that the primary information required by the user is color (as opposed to the intensity or the degree of saturation of the color) we have opted to present color in HSV (Hue, Saturation, Value) space, of which we use only the hue value at this stage. This color scheme was adopted by L. Cappelletti et. al. and J. N. Howard and represents colors as red, orange, yellow, green, blue, indigo, and violet (commonly memorized in the USA through the mnemonic ROY G BIV).

As in the previous color studies, we present hue through vibrotactile feedback. Unlike these studies, we do not provide feedback to the phalanges and hand. Vibrotactile feedback has been studied on numerous locations throughout the body, including the fingertips, wrist, forearm, chest, and neck [e.g. 9-10]. Kumagai and Hawes found that users preferred feedback to the torso and chest locations. Lam compared performance for vibrotactile feedback to the forearm and torso; performance was superior to the torso. Thus, we have chosen to provide vibrotactile feedback to the torso. It should be noted that the torso aligns with our goals of rendering the device unobtrusive to onlookers and taking advantage of a sensory system not actively used.

Our system is designed to map four color sensors to four vibrotactile actuators. So each tactor must be capable of encoding a range of colors, ideally ROY G BIV. Van Erp recommended varying timing and location in order to vary vibrotactile perception [11]. Since our four sensors map directly to the four tactors, we are varying the temporal information between vibration pulses in order to describe different colors.

\section{HARDWARE SYSTEM}

Figure 1 shows a block diagram of the tactile-to-color system, which we have named a Chromo-Haptic SensorTactor (CHST) device. It consists of a glove which has four color-sensing modules (one on the thumb tip, and one each on the fore, middle and ring fingertips). If the sensors interfere with the natural haptic feedback of the user (e.g. when reading Braille), the sensors could be placed on other locations on the body. These modules are interfaced to a microprocessor system (at this stage, a notebook PC is used, but an embedded system is planned). A set of vibrotactile actuators ("tactors") is also interfaced to the microprocessor system. The tactors are mounted on a soft elastic fabric belt which can be worn around the torso in such a way that the tactors are placed against the skin with moderate pressure.

A photograph of the physical components of the system is shown in Figure 2.

\section{A. Color Sensors}

The color sensors used are based on the Avago ADJD S371-QR999 RGB digital color sensor module. This is a miniature $(3.9 \times 4.5 \mathrm{~mm})$ surface-mount device which contains a white LED for illumination and four photodetectors - one each with blue, green, red, and wideband sensitivity.
It also contains a 10-bit integrating ADC with a digitally settable integration capacitor. The spectral response of the sensor is reproduced in Figure 3.

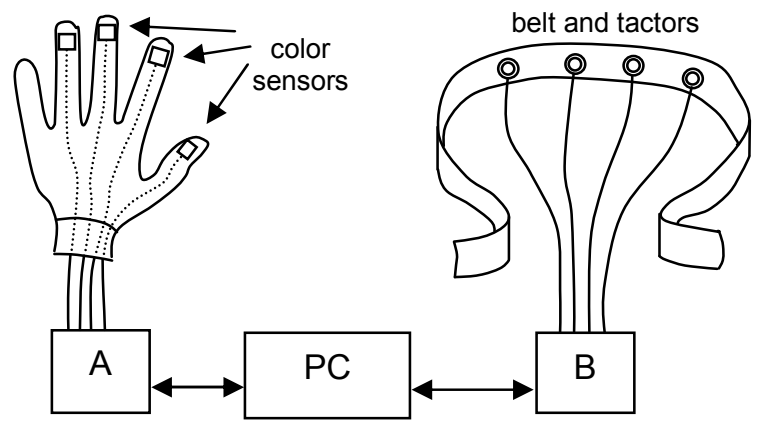

Figure 1. Block diagram of the CHST device. The individual color sensors are brought together in an interface (A) which doubles as a serial-USB converter. The data is analyzed by a software application running on the PC. The resulting haptic outputs are sent to an interface board (B) containing signal generators and power amplifiers, from which individual signals are sent to each tactor.

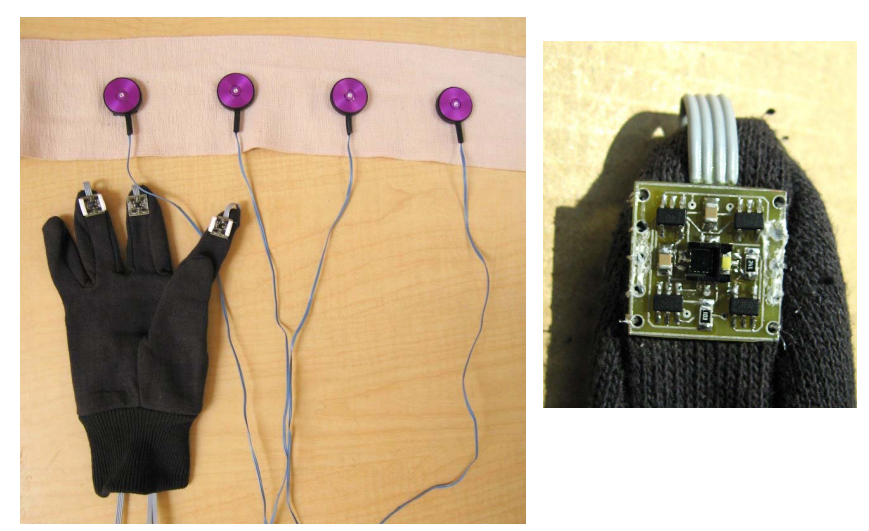

Figure 2. Left: the physical components of the CHST system, showing the glove with color sensors and the belt with tactor actuators. Right: detail view showing the fingertip PCB with an Avago sensor in the center and associated regulators and microprocessor around the periphery. The board is sewn onto the glove through the rows of holes on its vertical edge.

The Avago color sensors are mounted on a $15 \mathrm{~mm} \times 15 \mathrm{~mm}$ PCB, shown in Figure 2, which also contains SMD voltage regulators, a 6-pin microcontroller (PIC10F222) and a serial transceiver chip (MAX3323E). This produces a small standalone unit which can be used individually (interfaced directly to a $\mathrm{PC}$, for example) or combined in a sensor array.

\section{B. Tactors}

The tactors used are $\mathrm{C}-2$ vibrotactile transducer units supplied by EAI Inc. These have become widely used in vibrotactile research and provide a single point of vibratory stimulation in the frequency range $30-350 \mathrm{~Hz}$. The $\mathrm{C}-2$ tactors are interfaced to a PC via the EAI Eval 2.09 board, which provides signal generation and power amplification for up to eight tactors. 


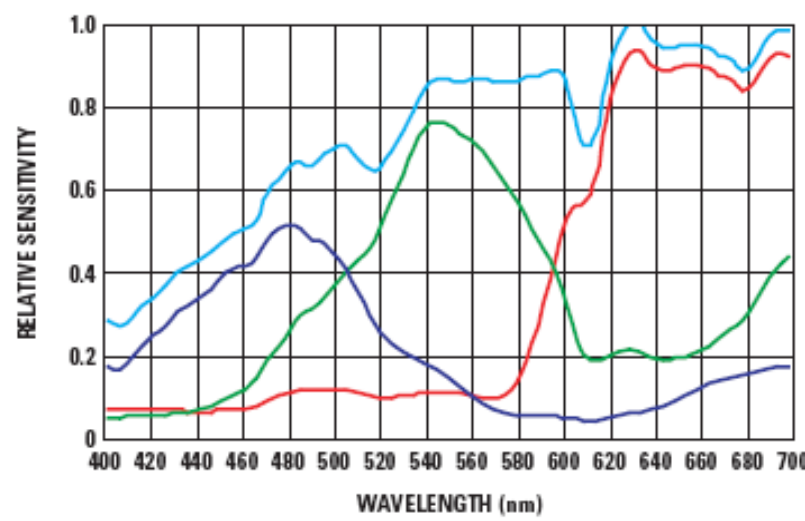

Figure 3. The spectral response of the Avago ADJD S371-QR999 color sensor (manufacturer's data). It can be seen that there is considerable overlap in the spectral sensitivities of the color specific photodetectors.

The tactors are mounted on a soft flexible belt using velcro; this mounting allows for easy variation of tactor spacing and mounting pressure during experiments. Both the color sensors and the tactors are controlled through Matlab ${ }^{\circledR}$ as serial devices.

\section{INTERFACING AND SOFTWARE}

\section{A. Color Conversion}

The Avago color sensors have two user-specifiable parameters, which are the integration time and the integration capacitor. In our system, these can be dynamically adjusted to provide an optimum balance of low noise and linear operation; however, in practice, adjustment was not necessary for indoor operation. The sensor returns four 10-bit variables, representing the red, green, blue and "clear" (broadband) components of received light. The software process required is shown diagrammatically in Figure 4.

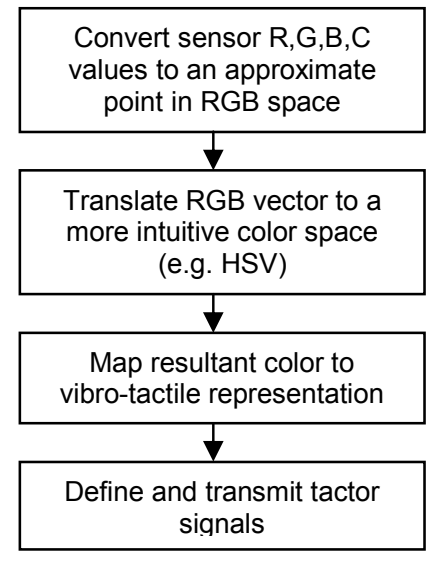

Figure 4. Elements of the software process.

The first step in the software process is non-trivial, given the combined photodiode responses shown in Figure 3. As can be seen, the highest response of the red detector relative to green and blue occurs at $630 \mathrm{~nm}$ and is reduced for longer wavelengths; it is also uncertain what the near-IR response of the diodes is. In addition, the presence of the white LED on

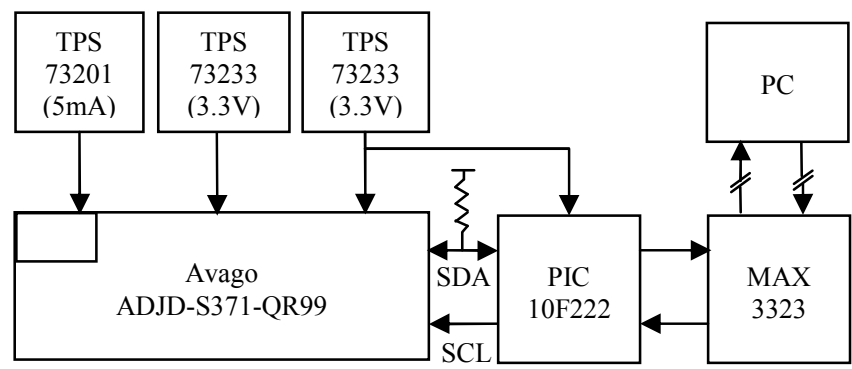

Figure 5. Block diagram of the color sensor, showing the simple arrangement of regulators, microprocessor, and serial communications interface.

the detector is occasionally problematic when highly reflective or internally illuminated objects are being scanned. We have developed a set of offset and scaling parameters that, in combination with some simple heuristics, give us a nominal RGB space which detects variations of red, yellow, green and blue with a high degree of accuracy.

As discussed in Section II, we transform the RGB color scheme to HSV using the standard Matlab function. This gives hue (the primary attribute of color) on a single closed axis or circle; i.e. the hue ranges from red $\left(\mathrm{H}=0\right.$ or $\left.0^{\circ}\right)$ through green $\left(\mathrm{H}=0.33\right.$ or $\left.120^{\circ}\right)$ to blue $\left(\mathrm{H}=0.67\right.$ or $\left.240^{\circ}\right)$ and back to red at $\mathrm{H}=1$, with mixtures of these primary colors occurring at intermediate values.

\section{RESULTS}

The use of a PC and Matlab ${ }^{\circledR}$ for the development work described here had many advantages, particularly in terms of rapid experimentation with color mappings. However, there was a significant latency (defined as the elapsed time between physical color change and the start of the tactile signal) experienced through the use of this platform. This latency should be reduced when the PC is replaced by an embedded system, as would be required in a commercial device. Typical latencies were of the order of $1.1 \mathrm{~s}$ for the color sampling routine and $0.3 \mathrm{~s}$ for the tactor output. Nonetheless, the system proved surprisingly easy to use. A number of mappings and tactile stimulation strategies were evaluated.

The standard evaluation method involved displaying random blocks of color onto an LCD screen, against which the subject was holding the relevant fingertip. With the free (ungloved) hand, the subject would press a key corresponding to the color perceived via tactile feedback. All the tests described below used nominal "primary" colors, i.e. red, green, blue, and yellow (while yellow is obviously not a standard primary color in the additive spectral sense, it is primary in a perceptual context).

The simplest mapping implemented was to use only one fingertip at any one time, and to map this to four tactors, each of which corresponded to one of the chosen colors. Upon sensing the color, the tactor would vibrate at a specified frequency and gain. It was found that this mapping was almost instantly learnable without any prior experience of tactor systems, and all users were able to perform with near $100 \%$ accuracy on their first attempt. Whilst this is not 
completely surprising, given the simplicity of the mapping, it was noted that the sensing felt completely intuitive. The only effort required in learning was to memorize the color-to-tactile mapping, develop a sense of the latency in the system, and move the fingertip at a speed and cadence that allowed for the latency.

A more complicated mapping required the use of two tactors to represent four colors; in other words two fingertips mapped to four tactors. The mapping used is shown in Table I. The tactors are numbered from the leftmost. during tests, the glove was worn on the right hand.

TABLE I. TACTILE MAPPING USED TO REPRESENT COLORS IN A TWOFINGER, FOUR COLOR SYSTEM.

\begin{tabular}{|c|c|c|c|c|}
\hline Color & Red & Green & Blue & Yellow \\
\hline Thumb & T2 & $\begin{array}{c}\mathrm{T} 1+\mathrm{T} 2 \\
\text { simultaneous }\end{array}$ & $\begin{array}{c}\mathrm{T} 1+\mathrm{T} 2 \\
\text { alternating }\end{array}$ & $\mathrm{T} 1$ \\
\hline Forefinger & $\mathrm{T} 4$ & $\begin{array}{c}\mathrm{T} 3+\mathrm{T} 4 \\
\text { simultaneous }\end{array}$ & $\begin{array}{c}\mathrm{T} 3+\mathrm{T} 4 \\
\text { alternating }\end{array}$ & $\mathrm{T} 3$ \\
\hline
\end{tabular}

It was possible to obtain $100 \%$ accuracy with this scheme, but only with significant latency; users also found a rapid onset of fatigue, as full concentration was required to attend to the signals from the desired fingertip in the presence of distracting signals from other fingers. A comparison of accuracy with latency is shown in Figure 6. It was noted by subjects that simultaneous stimulation from two adjacent tactors was particularly difficult to detect, and this is reflected in the larger error rate for green detection in this test.

The mapping for four fingertips to four tactors could be done in a number of ways. We mapped each finger to a single tactor and encoded the colors using sequential pulse codes (one finger at a time) arranged in the mapping shown in Table II (where "V" represents a vibration and "P" represents a pause, of approximately the same length). Results are shown in Figure 6.

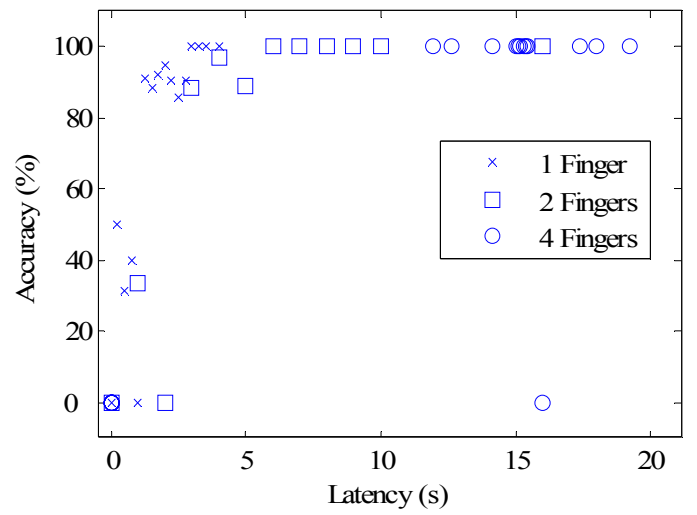

Figure 6. Results for the CHST device when used (at different times) for single, two and four-fingered operation, with the user attempting to balance latency with accuracy. Points represent ratio of correct/incorrect trials per latency bin. This was the first use by a typical user. Tactors were placed at

$150 \mathrm{~mm}$ intervals around the torso at navel level, symmetrical with the midline. It can be seen that a high degree of accuracy can be achieved with moderate latency. For multi-fingered operation, the longer latency is caused by waiting for the system to cycle through to the finger of interest.
TABLE II. TACTILE MAPPING USED TO REPRESENT COLORS IN A FOURFINGER, FOUR COLOR MAPPING

\begin{tabular}{|c|c|c|c|c|}
\hline Color & Red & Green & Blue & Yellow \\
\hline Output & V-V-V & V-P-V-V & V-V & V-V-P-V \\
\hline
\end{tabular}

\section{CONCLUSIONS}

We have developed the first complete color-to-tactile sensory substitution system. Its use is reasonably intuitive, and it required only a few minutes training for users to obtain accurate results. It was possible to map up to four colors per finger onto one tactor, using a pulse coded representation.

\section{FUTURE WORK}

We intend to develop a color classification scheme based on a support vector machine, which takes the four values as its inputs. This should allow us to identify more colors with reliability. In order to represent these colors, the system will be extended to eight tactors (which is possible without changing any other hardware).

\section{ACKNOWLEDGMENT}

The authors would like to thank the Institute for Neuromorphic Engineering and the Telluride Neuromorphic Engineering Workshop for support; and Anthony Lewis for helpful discussions about color representation.

\section{REFERENCES}

[1] L. Cappelletti, M. Feeri, and G. Nicoletti, "Vibrotactile colour rendering for the visually impaired within the VIDET project," Telemanipulator and Telepresence Technologies V, vol. 3524, pp. 9296, Nov. 1998.

[2] K. Kahol, J. French, L. Bratton, and S. Panchanathan, "Learning and perceiving colors haptically," Proceedings of the $8^{\text {th }}$ International ACM SIGACCESS Conference on Computers and Accessibility, pp. 173180,2006

[3] J.N. Howard, "Color Association with Differential Waveform Driven Force-Feedback Vibration Periodicities," (PhD Dissertation, Wichita State University, Submitted in 2007).

[4] J.C. Blish, M.H. Katcher, C.H. Rogers, and R.P. Shepard, "Optical-totactile image conversion for the blind," IEEE Transactions on Man Machine Systems, vol. 11, pp. 58-65, March 1970.

[5] Y. Yokokohji, R.L. Hollis, and T. Kanade, "Vision-based visual/haptic registration for WYSIWYF display," Proceedings of the 1996 IEEE/RSJ International Conference on Intelligent Robots and Systems, vol. 3, pp. 1386-1393, Nov. 1996.

[6] C. Sjöström, "Using haptics in computer interfaces for blind people," Conference on Human Factors in Computing Systems, pp. 245-256, 2001.

[7] S.W. Mereu and R. Kazman, "Audio enhanced 3D interfaces for visually impaired users," ACM SIGCAPH Computers and the Physically Handicapped, issue 57, pp. 10-15, Jan. 1997.

[8] P. Roth, L.S. Petrucci, A. Assimacopoulos, and T. Pun, "Audio-haptic internet browser and associated tools for blind and visually impaired computer users," Workshop on friendly exchanging through the net, March 2000.

[9] A. Lam, "Vibrotactile Pattern Recognition on the Torso with One and Two Dimensional Displays," (BS Thesis, MIT, Submitted in 2006).

[10] J.K. Kumagai and V.L. Hawes, "Assessment of Tactile Designs in Support of Wayfinding," Department of National Defence, Canada, Report DRDC Toronto CR-2005-021, 2005.

[11] J.B.F. van Erp, "Guidelines for the Use of Vibro-Tactile Displays in Human Computer Interactions," Eurohaptics, 2002. 